\title{
Um Relato de Experiência da Implantação de um Modelo de Fábrica de Software Escola (FaSEs)
}

\author{
Edmilson Barbalho Campos Neto ${ }^{1}$, Alba Sandyra Bezerra Lopes $^{1}$ e \\ Diego Silveira Costa Nascimento ${ }^{1}$ \\ ${ }^{1}$ Instituto Federal do Rio Grande do Norte \\ Campus Natal-Zona Norte \\ Natal - RN - Brasil \\ \{edmilson.campos, alba.lopes, diego.nascimento\}@ifrn.edu.br
}

\begin{abstract}
This paper presents an experience report of implantation of a software factory for education, named FaSEs, for students of technical and graduation course in computer at Instituto Federal do Rio Grande do Norte - Campus Natal Zona Norte. The goal was to create an environment where students could experience the stages of Software Engineering and still solve real demands. The methodology was applied inside classrooms and also in research projects. As results, $74.3 \%$ of students indicate that the learning process increased using the practical methods when compared with a theoretical approach.
\end{abstract}

Resumo. O presente artigo traz um relato de experiência da implantação de uma fábrica de software escola, denominada FaSEs, para os alunos dos cursos técnicos em Informática e Informática para Internet e curso superior em Licenciatura em Informática do Instituto Federal do Rio Grande do Norte - Campus Natal-Zona Norte. O objetivo foi criar um ambiente para que os alunos pudessem vivenciar de forma tangível as fases da Engenharia de Software e ao mesmo tempo atender a demandas reais. A metodologia foi aplicada tanto dentro de sala de aula como em projetos de pequisa. Como resultado, 74,3\% dos alunos indicaram ter absorvido melhor os conteúdos através da experiência de vivência na fábrica, ao comparar com a metodologia tradicional de aulas expositivas.

\section{Introdução}

O conceito Fábrica de Software (FS) foi usada pela primeira vez na década de 60, mas popularizada apenas nos anos 90. Segundo [Herbsleb and Grinter 1999], pode ser definido como "um processo estruturado, controlado e melhorado de forma contínua, considerando abordagens de engenharia industrial, orientado para o atendimento a múltiplas demandas de natureza e escopo distintas, visando à geração de produtos de software, conforme os requerimentos documentados dos usuário e/ou clientes, da forma mais produtiva e econômica possível".

No âmbito acadêmico, a FS é uma proposta inovadora que busca a interação concomitante entre teoria e prática, subsidiando os alunos na aplicação real dos conceitos aprendidos em sala de aula, ao mesmo tempo que prepara mão de obra especializada para atuar no mercado de desenvolvimento de software. O modelo vem sendo seguido em instituições de educação do Brasil e no Mundo. Por exemplo, no 
trabalho de [Brito et al. 2013], os autores apresentaram uma proposta de criação de uma metodologia para FS no Instituto Federal de Educação, Ciência e Tecnologia de Goiás - campus Inhumas de forma a atender demandas reprimidas de desenvolvimento de software, bem como, proporcionar aos alunos experiências práticas. Já no trabalho de [Oliveira and Colenci 2003], os autores descrevem um relato de experiência da implantação de uma FS acadêmica implantada na Faculdade de Tecnologia de Jundiaí em São Paulo que visa estimular a capacitação tecnológica dos alunos e o incentivo a criação de empresas competitivas. No trabalho de [Siqueira et al. 2008], os autores apresentaram uma abordagem metodológica para o ensino de Engenharia de Software (ES) na Escola Politécnica da Universidade de São Paulo, de forma que o ensino não ficasse restrito à sala de aula e o laboratório, mas que os alunos pudessem vivenciar um processo de desenvolvimento de software. Enquanto que no trabalho de [Borges et al. 2012], os autores relatam as experiências alcançadas no projeto de extensão de uma FS desenvolvido no Instituto Federal de Educação, Ciência e Tecnologia do Rio Grande do Sul, que teve por objetivo propiciar aos alunos capacitação nas principais tecnologias de mercado e vivência no mundo do trabalho. E o trabalho de [Ahmad et al. 2014], que os autores fizeram uma avaliação da percepção dos alunos através da utilização de quadro Kanban e aprendizado colaborativo que foi desenvolvido na Universidade de Oulu na Finlândia.

De acordo com as experiências anteriormente citadas no que se refere à aplicação do conceito de FS em âmbito formador, o presente artigo parte da hipótese de que tal abordagem é promissora na formação dos alunos na disciplina de ES permitindo, principalmente, um amadurecimento técnico e profissional.

De forma a comprovar nossa hipótese, foi desenvolvido o projeto de extensão $\mathrm{Fa}$ SEs, um acrônimo para Fábrica de Software Escola. O projeto tem como objetivo geral possibilitar aos estudantes dos cursos da área de tecnologia e afins do Instituto Federal do Rio Grande do Norte - Campus Natal-Zona Norte, aplicar os conhecimentos de ES trabalhados em sala de aula para criação de soluções reais demandadas pela própria comunidade escolar ou industrial. Com isso, almeja-se possibilitar aos alunos uma vivência de um ambiente mais próximo da realidade de uma organização de desenvolvimento de software, que permite [Herbsleb and Grinter 1999]: capacitar os alunos para a compreensão e resolução de problemas relacionados à produção de software, seguindo processos de desenvolvimento de sistemas; pesquisar e aplicar novas tecnologias e metodologias de desenvolvimento de software; divulgar o potencial dos alunos participantes junto ao mercado de trabalho, com vista a obtenção de colocações dentro das empresas de desenvolvimento de software; dar suporte a projetos da instituição, fornecendo soluções de software personalizadas; e promover outras ações (palestras, cursos de formação complementar, certificação profissional, eventos, entre outros).

Esse artigo está organizado da seguinte forma. A Seção 2 apresenta as metodologias de ensino e desenvolvimento utilizadas pela FaSEs. A Seção 3 apresenta e discute os resultados alcançados e Seção 4 apresenta uma avaliação da metodologia adotada. Por fim, o artigo é concluído na Seção 5, onde são realizadas as considerações finais.

\section{Metodologia de Ensino de Engenharia de Software na FaSEs}

Segundo [Sommerville 2011], ES é uma disciplina cujo foco está em todos os aspectos da produção de software, desde os estágios iniciais da especificação do sistema até sua 
manutenção. Embora não exista um consenso entre autores quanto a terminologia adotada para as fases de desenvolvimento de um projeto de software, neste trabalho adotamos as etapas categorizadas como: levantamento de requisitos, análise, projeto, implementação e implantação.

\subsection{Seleção e Acompanhamento de Projetos}

A metodologia adotada para o desenvolvimento deste projeto buscou envolver os alunos nos diversos anos de ensino dos cursos técnicos e em Informática e Informática para Internet e do curso superior em Licenciatura em Informática da instituição. Para tanto, a FaSEs foi organizada em dois grupos, aqui chamados de divisões da fábrica. A primeira, a Divisão Acadêmica (DA), abrange os projetos executados diretamente em sala de aula, pelos problemas e alunos envolvidos nas disciplinas integradas, sendo esses, portanto, projetos curriculares. O segundo grupo, intitulado Divisão de Inovação (DI) compreende os projetos executados por editais de pesquisa de fomento interno ou externo da instituição, seja esses por meio de bolsas de pesquisa ou não.

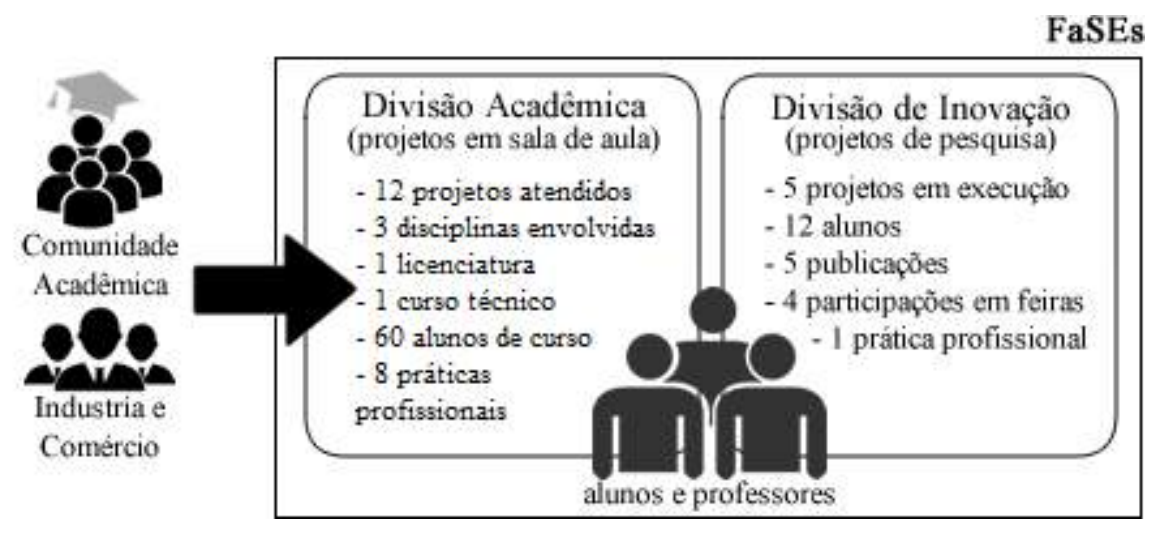

Figura 1. Visão geral dos resultados.

A Figura 1 ilustra uma visão geral da estrutura da FaSEs com alguns números correspondentes ao cenário atual de sua implantação na instituição. Além disso, é possível observar no lado esquerdo da figura, os grupos responsáveis por demandar soluções a serem atendidas por cada uma das divisões da fábrica, a depender da natureza da execução do projeto (acadêmica ou pesquisa). Independente da divisão responsável, todos os projetos da FaSEs atendem necessariamente a soluções que surgem desses grupos de demandantes (stakeholders), a seguir descritos:

- Comunidade Acadêmica (interno): Grupo formado pelos alunos, professores e servidores da própria instituição; as soluções demandadas por esse grupo buscam otimizar/corrigir problemas diretamente ligados ao dia a dia da instituição; e

- Indústria e Comércio (externo): Grupo formados por stakeholders diretamente ligados a indústria ou comerciantes locais, que demandam soluções específicas para o seu negócio.

Pela necessidade de gerenciar todos os projetos de forma unificada, adotou-se a ferramenta Trello ${ }^{1}$ que possibilita criar quadros de tarefas específicos (Kanban Board)

\footnotetext{
${ }^{1}$ http://www.trello.com
} 
para cada projeto e associar alunos e professores participantes. Além dessa ferramenta, foi adotado um repositório de código visando melhor versionamento dos sistemas e possibilitando que os projeto possam ser continuados por outras equipes no futuro. No primeiro ano de implantação, os projetos foram disponibilizados no GitHub ${ }^{2}$. No segundo ano, o repositório adotado foi o GitLab ${ }^{3}$.

\subsection{Processo de Desenvolvimento de Software}

O processo seguido pela FaSEs baseia-se no OpenUP ${ }^{4}$, um modelo interativo-incremental dividido em quatro fases: Concepção, Elaboração, Construção e Implantação. Cada fase foi subdividida em iterações, com duração média de 15 dias, e ao final de cada iteração são agendadas entregas para cada equipe apresentar os avanços obtidos. As entregas funcionavam como entregas simuladas de releases parciais da solução aos clientes/demandantes, que em algumas projetos participaram ativamente do processo, com avaliações das entregas. A escolha do processo OpenUP se deu pelo fato dessa metodologia ser capaz de fomentar a prática com profundidade de todas as atividades da ES, desde a análise à implantação, no ambiente acadêmico.

A Tabela 1 apresenta as principais atividades e tarefas gerados pela primeira fase do processo, a Concepção. Extraída do OpenUP, o objetivo dessa fase é identificar e abordar os stakeholders (interessados) do sistema para extrair/entender a relação de requisitos funcionais e não funcionais que o projeto deverá atender, gerando, para tanto, alguns artefatos específicos para este fim.

Tabela 1. Artefatos gerados na fase de Concepção do processo

\begin{tabular}{|l|l|l|}
\hline \multicolumn{1}{|c|}{ Atividade } & \multicolumn{1}{c|}{ Tarefa } & \multicolumn{1}{c|}{ Artefato gerado } \\
\hline Iniciar projeto & Iniciar projeto & $\begin{array}{l}\text { - Documento de Visão } \\
\text { - Glossário }\end{array}$ \\
\hline Planejar e Gerenciar Iteração & Planejar iteração & $\begin{array}{l}\text { - Plano de Riscos } \\
\text { - Plano de Iteração }\end{array}$ \\
\hline \multirow{2}{*}{ Identificar Requisitos } & $\begin{array}{l}\text { Identificar requisitos } \\
\text { Detalhar cenários de UDC } \\
\text { Realizar protótipos de telas }\end{array}$ & $\begin{array}{l}\text { - Tabela de RNF } \\
\text { - Casos de Uso (CDU) } \\
\text { - Protótipos baixa fidelidade }\end{array}$ \\
\hline Esboçar arquitetura & Definir arquitetura do sistema & - Documento de arquitetura \\
\hline
\end{tabular}

A segunda fase do processo OpenUP, adaptada para o processo da FaSEs, tem o nome de Elaboração e seu objetivo é definir como as funcionalidades do sistema, identificadas na fase anterior, serão implementadas na fase de Construção seguinte. Para isso, é necessário identificar quais casos de uso apresentam maior risco para que possam ser implementados nessa fase, com o objetivo de validar a arquitetura projetada para o sistema. Considera-se casos de uso de maior risco (CDUMR) aqueles que, se não implementados/entregues ao clientes, desconfiguram a essência do sistema. A Tabela 2 reporta os artefatos gerados a fase de Elaboração.

As duas últimas fase do processo, a Construção e a Transição, têm como objetivo, respectivamente, implementar todos os casos de uso restantes e previstos para o sistema;

\footnotetext{
${ }^{2}$ http://www.github.com/fases

${ }^{3}$ http://www.gitlab.com

${ }^{4}$ http://epf.eclipse.org/wikis/openup/
} 
Tabela 2. Artefatos gerados na fase de Elaboração do processo

\begin{tabular}{|l|l|l|}
\hline \multicolumn{1}{|c|}{ Atividade } & \multicolumn{1}{c|}{ Tarefa } & \multicolumn{1}{c|}{ Artefato gerado } \\
\hline \multirow{2}{*}{ Gerenciar requisitos } & $\begin{array}{l}\text { Encontrar e descrever requisitos } \\
\text { Detalhamento de requisitos } \\
\text { Criar caso de teste }\end{array}$ & $\begin{array}{l}\text { - Especificação de CDUMR } \\
\text { - Plano de teste }\end{array}$ \\
\hline \multirow{2}{*}{ Definir arquitetura } & $\begin{array}{l}\text { Desenvolver arquitetura } \\
\text { Projetar banco de dados }\end{array}$ & $\begin{array}{l}\text { - Diagrama de classes } \\
\text { - Diagrama de pacotes } \\
\text { - MER }\end{array}$ \\
\hline \multirow{2}{*}{ Desenvolver um incremento da solução } & $\begin{array}{l}\text { Projetar a solução } \\
\text { Implementar a solução } \\
\text { Executar testes }\end{array}$ & $\begin{array}{l}\text { - Protótipos alta fidelidade } \\
\text { - Release com incremento } \\
\text { - Relatórios de testes }\end{array}$ \\
\hline Planejar e Gerenciar Iteração & Planejar interação & - Plano da Iteração \\
\hline
\end{tabular}

e validar e implantar o sistema em ambiente real. Na Construção, os casos de uso especificados nas fases anteriores são em plenitude implementados seguindo a arquitetura validada na fase de Elaboração. Esses casos de uso são validados por meio de testes durante o desenvolvimento e, ao final, transferidos para um ambiente real de produção, para utilização e aprovação por parte dos stakeholders do sistema.

\section{Resultados}

Esta seção apresenta os resultados obtidos pela FaSEs após dois anos da sua implantação no campus da instituição de ensino, de acordo com a natureza da divisão da fábrica (acadêmica ou inovação).

\subsection{Experiência de interdisciplinaridade}

Os projetos da Projetos da Divisão Acadêmica (DA) foram executados englobando duas disciplinas da estrutura curricular do quarto ano do curso técnico integrado em Informática: Programação para Internet (PPI) e Projeto de Desenvolvimento de Software (PDS). O projeto pedagógico do curso considera que as duas disciplinas possam desenvolver projeto integrador, de forma a possibilitar melhor consolidação dos conteúdos e objetivos das disciplinas. A ementa da disciplina de PDS busca proporcionar ao aluno a compreensão do processo de desenvolvimento de software, as atividades técnicas e as iterações envolvidas neste processo. Já a disciplina de PPI propõe que os alunos possam desenvolver aplicações com programação do lado do cliente e do servidor e controlar o estado e o acesso em aplicações na internet.

Em 2015, buscando atingir esses objetivos, os professores dividiram a turma composta por 27 alunos em seis equipes e discutiram as demandas de sistemas existentes na fábrica. Das seis demandas apresentadas na turma, três eram da comunidade acadêmica e três da indústria e comercio. Cada equipe ficou responsável por uma demanda específica. Além disso, no que concerne a execução das atividades e da interdisciplinaridade, a disciplina de PDS foi a responsável por conduzir o processo de desenvolvimento, agregando as fases do processo e as atividades específicas de implementação trabalhadas com alunos pelo professor da disciplina de PPI. Em 2016, igual divisão foi realizada com uma nova turma formada por 31 alunos concluintes.

\subsection{Experiência entre cursos de níveis distintos}

Além da integração das disciplinas intracurso ocorrida no nível técnico em 2015, no segundo ano da vivência da FaSES da Divisão Acadêmica, em 2016, foi viabilizada a 
integração de disciplina de cursos diferentes e de níveis distintos. A experiência contou com a integração da disciplina de Engenharia de Software (ES) ofertada a uma turma de curso superior em licenciatura plena em informática com as duas disciplinas de PPI e PDS do curso técnico já integradas no ano anterior. Nessa nova formatação, os alunos de nível superior em licenciatura atuavam como mentores dos projetos, acompanhando a vivência do projeto e dando suporte pedagógico necessário ao entedimento dos conceitos envolvidos na execução de um processo de desenvolvimento de software, enquanto os alunos do curso técnico atuavam na implementação e geração dos artefafos previstos no processo.

\subsection{Projeto da Divisão Acadêmica (DA)}

A Tabela 3 apresenta a distribuição dos projetos. Para cada projeto é indicado o ano de início, o tipo do projeto, o objeto/área de concentração, a origem da demanda (comunidade acadêmica - CA ou industria e comércio - IC) e a quantidade de alunos envolvidos em cada equipe. Além disso, a tabela também apresenta a quantidade de trabalhos que foram utilizados como trabalho de conclusão de curso pelos alunos do quarto ano, concluintes do curso técnico em Informática. A seguir, são apresentados dois projetos de naturatezas distintas para exemplificar a diferença entre os tipos de demandantes da FaSEs na Divisão Acadêmica. Mais informações sobre os projetos desenvolvidos podem ser obtidas através do site do projeto ${ }^{5}$.

Tabela 3. Sumário dos resultados da Divisão Acadêmica da FaSEs

\begin{tabular}{|l|c|c|c|c|c|c|}
\hline \multicolumn{1}{|c|}{ Projeto } & Ano & Tipo de projeto & Objeto & Demandante & \#alunos & \#TCC \\
\hline Brainzer & 2015 & Jogo educativo & Saúde & IC $^{2}$ & 5 & 3 \\
\hline Comunica & 2015 & SI $^{3}$ & Comunicação & CA $^{4}$ & 5 & 1 \\
\hline Emprenet & 2015 & Serviço online & Domésticas & IC & 3 & - \\
\hline Filmões & 2015 & Serviço online & Filmes & IC & 4 & - \\
\hline SUSI & 2015 & SI & Saúde & CA & 5 & - \\
\hline WorldShare & 2015 & Rede social & Acadêmico & CA & 5 & - \\
\hline ACHA & 2016 & SI & Carga horária & IC & 5 & 3 \\
\hline COLINFO & 2016 & Serviço online & Publicações & CA & 5 & 3 \\
\hline BinChallenger & 2016 & Jogo educativo & Binários & IC & 5 & 3 \\
\hline FaSEs Web & 2016 & SI & FaSEs & IC & 5 & 1 \\
\hline MudiFast & 2016 & SI & Resoluções & CA & 1 & 3 \\
\hline SIGMIN & 2016 & SI & Minicursos & CA & 6 & 6 \\
\hline
\end{tabular}

(1) TCC - Trabalho de Conclusão de Curso; (2) IC - Indústria e Comércio; (3) SI - Sistema de Informação; (4) CA Comunidade Acadêmica

O projeto Brainzer (IC) teve como objetivo o desenvolvimento de um portal de jogos online para auxiliar portadores da doença Alzheimer no estímulo de sua cognição. No processo de desenvolvimento, a equipe realizou entrevistas com médico especialista, membros de associações de auxílio a familiares e portadores da doenças. Os jogos basearam-se em atividades endossadas por especialistas nesse tipo de doença neurodegenerativa, como jogos da memória, caça-palavras e diversos questionários intuitivos.

O projeto Worldshare (CA), por sua vez, foi desenvolvido para atender uma demanda institucional externada pelos docentes. A demanda foi por uma ferramenta onde os alunos pudessem compartilhar suas produções acadêmicas e culturais, tais como poesias, crônicas, além de resumos e artigos técnicos de disciplinas curriculares. Antes

\footnotetext{
${ }^{5}$ http://fases.ifrn.edu.br/
} 
da publicação do conteúdo, a produção passa pela revisão de um professor da área, que aponta trechos carentes de melhorias que são corrigidos pelo aluno.

\subsubsection{Utilização dos projetos como prática profissional}

Além da utilização dos projetos para a consolidação dos conteúdos das disciplinas de PDS e PPI, os alunos do curso técnico participantes dos projetos da Divisão Acadêmica ainda puderam aproveitar a vivência extra-curricular possibilitada pelos projetos desenvolvidos para integralização da carga horária de prática profissional prevista no projeto pedagógico do curso técnico na instituição, pré-requisitio para conclusão de curso, contribuindo assim para melhoria dos índices de êxito do curso.

Para avaliar esse impacto fomentado pela Divisão Acadêmica nos índices de conclusão, foram comparados os índices acadêmicos do ano letivo de 2015 e 2016 (após dois ano de implantação da FaSEs) com os de 2014 (ano anterior). A análise focou na avaliação da Taxa de conclusão (TC) de curso em cada ano letivo, que considera apenas os alunos que integralizaram todos os componentes curriculares, incluindo a prática profissional, no ano letivo analisado. A Tabela 4 apresenta um sumário desa análise comparativa.

Tabela 4. Resultado da análise comparativa

\begin{tabular}{|l|c|c|c|c|}
\hline Ano letivo & \#turmas & \#alunos & \#TC $^{1}$ & \#TC (FaSEs) $^{\text {ThC }}$ \\
\hline 2014 & 2 & 49 & $4 \%(2)$ & - \\
\hline 2015 & 1 & 27 & $79 \%(23)$ & $24 \%(7)$ \\
\hline 2016 & 1 & 31 & $100 \%(31)$ & $61 \%(19)$ \\
\hline
\end{tabular}

(1) Taxa de conclusão

Após essa análise, foi possível observar um avanço significativo na Taxa de Conclusão entre os anos de 2014 e 2015, que aumentou de 4\% (apenas dois alunos em 2014) para 79\% em 2015 (23 alunos) e, em 2016, atingiu 100\% de conclusão. Dos 23 alunos que concluíram suas práticas profissionais em 2015, sete utilizaram projetos desenvolvimento pela FaSEs, o que implica uma Taxa de Conclusão relacionada a FaSEs de 24\%. Esse número teve um crescimento ainda mais expressivo no segundo ano do projeto, atigindo $61 \%$, o que equivale a 19 alunos.

\subsection{Projetos da Divisão de Inovação (DI)}

Os projetos desenvolvidos nessa divisão atuam numa outra frente de resultados, responsável por impulsionar a produção científica da FaSEs. Os projetos, diferente dos da divisão acadêmica, que são desenvolvidos em sala de aula, são fomentados por editais de pesquisa internos ou externos e todos com potencial de inovação. Nesse sentindo, os resultados desses projetos podem ser mensurados nos número de publicações científicas e acadêmicas culminadas por cada um deles. A Tabela 5 a seguir apresenta um sumário desses resultados por projeto nesse biênio de implantação da metodologia proposta pela FaSEs, além de informações complementares sobre a origem de algumas das demandas de cada projeto e quantidade de alunos envolvidos por equipe.

O projeto Epuppy foi o projeto pioneiro da FaSEs e surgiu de uma demanda proveniente de uma clínica veterinária local, que externou, a partir da vivência diária, a 
Tabela 5. Sumário dos resultados da Divisão de Inovação

\begin{tabular}{|l|c|c|c|c|c|c|}
\hline \multicolumn{1}{|c|}{ Projeto } & Demandante & \#alunos & \#feiras & \#publicações & \#premiações & \#TCC $^{T}$ \\
\hline EPuppy & IC $^{2}$ & 5 & 1 & 1 & 0 & 3 \\
\hline Luzômetro & CA $^{3}$ & 3 & 0 & 1 & 0 & 0 \\
\hline SmartGás & IC & 2 & 3 & 2 & 2 & 0 \\
\hline TED Saúde & IC & 2 & 5 & 1 & 4 & 2 \\
\hline FreeAccess & IC & 3 & 0 & 1 & 1 & 0 \\
\hline PocketChef & IC & 2 & 0 & 1 & 0 & 0 \\
\hline
\end{tabular}

(1) TCC - Trabalho de Conclusão de Curso; (2) IC - Indústria e Comércio; (3) CA - Comunidade Acadêmica

necessidade de reunir em uma única rede as informações acerca dos animais domésticos e todos os envolvidos no cuidado desses animais, possibilitando a identificação do animal através de um QR-Code, que pode ser acoplado à coleira do animal.

O projeto SmartGás (Figura 2) surgiu de um potencial de inovação da industria e comércio de gás de cozinha doméstico. O projeto propõe o desenvolvimento e plataforma inteligente para o controle do consumo do gás. A plataforma fornece informações em tempo real da quantidade de gás restante no botijão, além de dados essenciais para controle da economia doméstica [Medeiros et al. 2016]. O projeto, com publicações nacionais e internacionais, é financiado por edital interno e conta com dois alunos do terceiro ano do curso técnico em informática para internet.
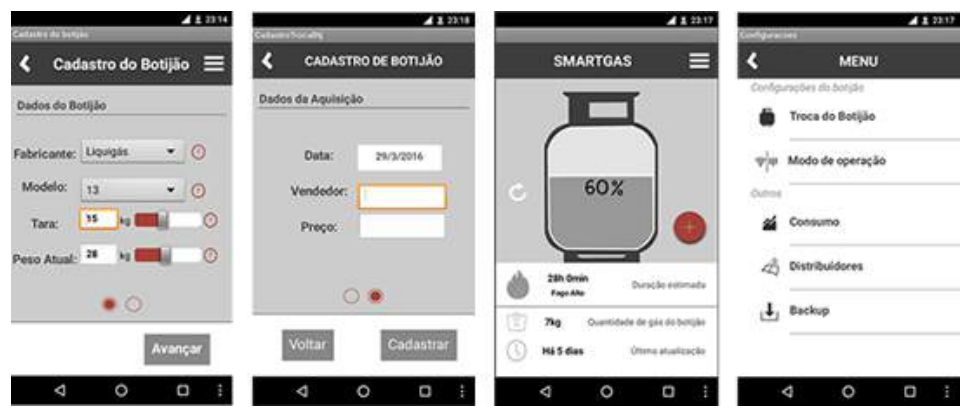

Figura 2. Protótipo do aplicativo desenvolvido no projeto SmartGás.

Outro projeto em destaque da FaSEs, o TEDSáude, propõe o desenvolvimento de uma plataforma educativa digital, e também visa atender um potencial de inovação do setor de saúde [Silva et al. 2016]. No projeto é desenvolvida uma plataforma interativa utilizando o dispositivo Kinect, a ser implantado nas salas de espera das unidades básicas de saúde, para a promoção da saúde. Os resultados do projeto foram disseminados por meio da publicação em eventos científicos e feiras tecnológicas, algumas das quais renderam premiações e credenciamentos para participação em outras feiras nacionais e internacionais.

\section{Avaliação dos Resultados}

Com o objetivo de avaliar a metodologia-modelo implantada pela FaSEs na Divisão Acadêmica, foi aplicada um questionário ao final da execução do segundo ano do projeto, que tinha como eixos principais: (i) Avaliar a compreensão/absorção dos conteúdos de engenharia de software; e (ii) Avaliar a perceptividade da metodologia adotada. A aplicação 
contou com 35 respondentes anônimos, entre alunos do curso técnico e do curso superior em licenciatura em informática, que participaram de projetos da Divisão Acadêmica durante o ano letivo de 2016.

Na primeira parte do questionários (primeiro eixo), os alunos foram perguntados sobre: (i) a nomeclatura e ordem das fases do processo adotado; e (ii) os objetivos de cada uma das fases. O objetivo era identificar se a adoção de um metodologia prática para ensino de engenharia de software foi eficiente para absorção dos conhecimentos teóricos da disciplina. $\mathrm{O}$ índice de acerto dos respondentes sobre a primeira fase (Concepção) foi de $100 \%$ e de $94,3 \%$ para a segunda fase (Elaboração). Já em relação das duas últimas fases, os índices de acertos foram um pouco menores. Sobre a fase de Construção, 74,3\% acertaram o seu objetivo, enquanto $77,1 \%$ responderam corretamente sobre o objetivo da fase de Transição.

Na segunda parte do questionário (segundo eixo), os alunos foram questionados sobre a experiência da vivência de um processo de engenharia de software na prática. 74,3\% (ou seja, 26 alunos), disseram acreditar que a vivência de um processo de engenharia de software na prática possibita alta contribuição dos conceitos teóricos da disciplina, enquanto $14,3 \%$ (5), responderam moderada contribuição. Apenas 4 respondentes disseram acreditar que aprenderiam mais com aulas expositivas do que uma vivência prática. A resposta dessa questão pode ser vista na Figura 3.a.

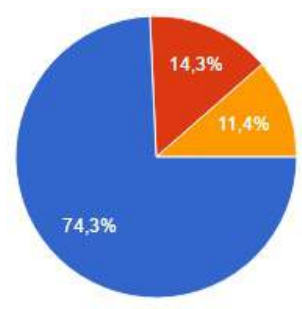

(a)

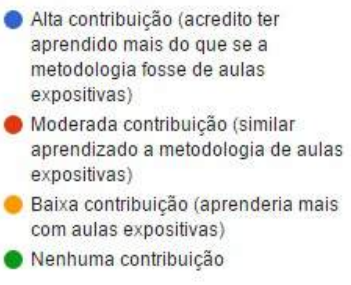

- Nenhuma contribuição

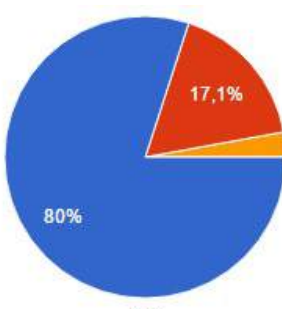

(b)

o esforço desprendido pela metodologia prática è MAIOR que numa metodologia tradicional de a.

- o esforço desprendido pela metodologia prática é MAIOR que numa metodologia tradicional de a o esforço desprendido pela metodologia prática é MENOR que... o esforço desprendido pela metodologia prática é MENOR que

\section{Figura 3. Nível de satisfação dos alunos com o estágio final dos seus projetos}

Também foi questionado a opinião dos alunos sobre a relação entre o esforço despendido e o aprendizado obtido na adoção de uma metodologia prática em relação a metodologias tradicionais de aulas expositivas-teóricas (Figura 3.b). Para 80\% (28) dos respondentes, o esforço de uma metodologia prática é maior, mas em compensação o aprendizado obtido também é maior, enquanto apenas $17,1 \%$ (6) acreditam ser maior o esforço e menor o aprendizado. Um único respondente afirmou ainda acreditar que um metodologia prática, além de possibilitar maior aprendizado, ainda requer um menor esforço que metodologias expositivas.

Sobre a satisfação geral com a metodologia prática adotada na disciplina de PDS e PPI, 68,5\% (24) responderam excelente ou boa, enquanto $25,7 \%$ (9) indicaram como regular, apontando como necessidade de melhoria maior tempo de acompanhamento dos projetos em sala de aula, uma limitação da carga horária das disciplinas envolvidas. Por fim, foi solicitado que os alunos indicassem o nível de satisfação com o estágio final dos seus respectivos projetos, numa escala de 1 a 5 , e mais de $80 \%$ responderam entre 4 e 5 .

Além desses resultados, é possível avaliar o impacto da Divisão de Inovação pelo número de publicações (5), premiações (4) e projetos (4) já apresentados na Seção 4.2. 
Nos próximos anos será possível comparar também esses números para essa divisão de modo a observar a evolução desse indicadores em face aos demais projetos da instituição.

\section{Considerações Finais}

Este trabalho apresentou um relato de experiência da implantação de uma Fábrica de Software Escola, denominada FaSEs, em uma instituição de ensino. A execução do projeto culminou no desenvolvimento bem sucedido de 18 projetos de software no ambiente acadêmico. Nesse período, os alunos puderam vivenciar as etapas de concepção, elaboração, construção e implantação inerentes a um processo de desenvolvimento de software. Como consequência, a implantação da fábrica contribuiu para a maturidade dos alunos no processo de desenvolvimento de sistemas. Ao serem questionados, $80 \%$ alunos consideraram que, apesar do esforço despendido na condução da disciplina de maneira prática ser maior, o aprendizado é também maior. E $80 \%$ dos alunos consideraram como satisfatório o estágio final dos seus projetos. Como trabalhos futuros, pretende-se incluir as etapas de testes e manutenção na metodologia de ensino para que alunos possam vivenciar também o processo de continuidade de um sistema já em desenvolvimento.

\section{Referências}

Ahmad, M. O., Liukkunen, K., and Markkula, J. (2014). Student perceptions and attitudes towards the software factory as a learning environment. In IEEE Global Engineering Education Conference, pages 422-428.

Borges, K. S., de Carvalho, T. P., and de Moraes, M. A. C. (2012). Programa de extensão "fábrica de software acadêmica": contribuindo para a formação profissional na área da informática. Workshop sobre Educação em Computação.

Brito, M. C. A., Silva, F. P., and Cabral, E. P. (2013). Elaboração de uma metodologia de desenvolvimento de software para a fábrica de software de uma instituição de ensino. Revista Brasileira de Informática na Educação, 21(2):52-61.

Herbsleb, J. D. and Grinter, R. E. (1999). Splitting the organization and integrating the code: Conway's law revisited. In International Conference on Software Engineering, pages 85-95.

Medeiros, G. V. S., Santos, M. R., Lopes, A. S. B., and Campos Neto, E. B. (2016). Smartgás: uma plataforma inteligente para monitoramento do consumo de gás de cozinha. In VIII Computer on the Beach, Florianópolis, SC.

Oliveira, D. H. and Colenci Neto, A. (2003). Fábrica de software: Promovendo a criação de empresas competitivas em tecnologia da informação. XXIII Encontro Nacional de Engenharia de Produção.

Silva, F. M., Lopes, A. S. B., and Campos Neto, E. B. (2016). Uma aplicação de terminais interativos de interface natural em ambientes inteligentes. In XV Simpósio Brasileiro sobre Fatores Humanos em Sistemas Computacionais (IHC), São Paulo, SP.

Siqueira, F. L., Barbarán, G. M. C., and Becerra, J. L. R. (2008). A software factory for education in software engineering. In IEEE 21st Conference on Software Engineering Education and Training, 2008, pages 215-222.

Sommerville, I. (2011). Engenharia de Software. Pearson, 9 edition. 